\title{
Avaliação da terapêutica em pacientes de alto risco cardiovascular
}

\section{Therapeutic evaluation in high-risk cardiovascular patients}

Juliana Gomes Freire ${ }^{1}$. Ricardo Pereira Silva ${ }^{1}$.

1 Hospital Universitário Walter Cantídio (HUWC), Fortaleza, Ceará, Brasil. 2 Universidade Federal do Ceará (UFC), Fortaleza, Ceará, Brasil.

\section{RESUMO}

Segundo o estudo Global Burden of Diseases, para o ano de 2020, a doença cardiovascular (DCV) permanecerá como a principal causa de óbito e incapacidade em ambos os sexos, no Brasil e no mundo. Em nosso país, as DCV são responsáveis por alta frequência de internações, ocasionando altos custos médicos e socioeconômicos. Daí a necessidade de se melhorar a abordagem desses pacientes. Objetivo: registrar a prática clínica vigente na prescrição das intervenções farmacológicas baseadas em evidência. Metodologia: estudo observacional, transversal e descritivo desenvolvido entre os pacientes do HUWC, no período de abril de 2014 a maio de 2015, incluindo indivíduos de alto risco cardiovascular. Resultados: a faixa etária prevalente situouse entre 50 e 70 anos; $64,7 \%$ eram mulheres; $68,5 \%$ eram pardos e $24,7 \%$ brancos. Hipertensão arterial sistêmica, dislipidemia e diabetes mellitus constituíram os principais fatores de risco cardiovasculares. Cerca de $64,6 \%$ dos pacientes apresentavam pressão arterial sob controle. Conclusão: $\mathrm{O}$ uso da terapia otimizada se encontra aquém da ideal; a maior parcela da população em estudo atingiu a meta para controle de PA.

Palavras-chave: Hipertensão arterial. Infarto agudo do miocárdio. Diabetes mellitus tipo 2. Inibidores da enzima conversora de angiotensina. Hipolipemiantes. Doenças cardiovasculares.

\section{ABSTRACT}

According to the Global Burden of Diseases study, for the year 2020, cardiovascular disease (CVD) will remain the leading cause of death and disability in both sexes in Brazil and worldwide. In our country, CVD are responsible for high frequency of hospitalizations, causing high medical and socioeconomic costs. Hence the need to improve the approach of these patients. Objective: to record the current clinical practice in the prescription of evidence-based pharmacological interventions. Methodology: an observational, transversal and descriptive study developed among HUWC patients from April 2014 to May 2015, including individuals at high cardiovascular risk. Results: the prevalent age range was between 50 and 70 years; $64.72 \%$ were women; $68.51 \%$ were brown and $24.72 \%$ white. Systemic arterial hypertension, dyslipidemia and diabetes mellitus were the main cardiovascular risk factors. About $64.62 \%$ of the patients had blood pressure under control. Conclusion: The use of optimized therapy is far from ideal; the largest portion of the study population reached the goal for BP control.

Keywords: Hypertension. Myocardial Infarction. Diabetes Mellitus, Type 2. Angiotensin-Converting Enzyme Inhibitors. Hypolipidemic Agents. Cardiovascular diseases.

Autor correspondente: Juliana Gomes Freire, Rua Dr José Lourenço, 2270, Joaquim Távora, Fortaleza, Ceará. CEP: $60115-282$. Telefone: +55 85 99789-1208. E-mail: julianagomesf0@gmail.com

Conflito de interesses: Não há qualquer conflito de interesses por parte de qualquer um dos autores.

Recebido em: 14 Ago 2018; Revisado em: 23 Abr 2019; Aceito em: 23 Abr 2019. 


\section{INTRODUÇÃO}

De acordo com projeções do clássico estudo Global Burden of Diseases, de Murray e Lopez, para o ano de 2020, há indicações de que a doença cardiovascular (DCV) não só permanecerá como a principal causa de óbito, mas também representará a principal causa de incapacidade em ambos os sexos, tanto no Brasil quanto no mundo (racionalidade e métodos- registro da prática clínica em pacientes de alto risco cardiovascular $(\mathrm{ARCV}))$. Em nosso país, as DCV têm sido a principal causa de morte e são responsáveis por alta frequência de internações, ocasionando custos médicos e socioeconômicos elevados. ${ }^{1}$

A HAS (Hipertensão Arterial Sistêmica) é a condição mais comum vista nos cuidados primários e que leva a infarto do miocárdio, acidente vascular cerebral (AVC), falência renal e morte, se não detectada precocemente e tratada adequadamente. ${ }^{2}$ A HAS tem alta prevalência e baixas taxas de controle, e é considerada um dos principais fatores de risco (FR) modificáveis e um dos mais importantes problemas de saúde pública. ${ }^{1}$ Devido ao aumento da obesidade e ao envelhecimento da população, o impacto global da HAS está aumentado e projeta-se que até 2025 um terço da população mundial será afetada. ${ }^{3}$

O objetivo primordial do tratamento da hipertensão arterial é a redução da morbidade e da mortalidade cardiovasculares. Assim, os anti-hipertensivos devem não só reduzir a pressão arterial, mas também os eventos cardiovasculares fatais e nãofatais, e, se possível, a taxa de mortalidade. ${ }^{1}$

Existe uma clara associação entre HAS e doença arterial coronariana (DAC). A redução adequada da pressão arterial (PA) é fundamental para a prevenção de eventos relacionados à $\mathrm{DAC}$, independentemente do grupo de medicamentos antihipertensivos utilizados, objetivando-se metas de PA de $130 \mathrm{x}$ $80 \mathrm{mmHg}$. 4,5

O infarto agudo do miocárdio (IAM) constitui um dos maiores problemas de saúde pública nos países industrializados. Nos EUA, a doença coronariana é responsável por 53\% das mortes por morbidade cardiovascular, ocorrendo cerca de 780 mil casos de IAM por ano em americanos com idade acima de 65 anos. Anualmente no Brasil, estima-se cerca de 300 a 400 mil casos de IAM, tornando-se a principal causa isolada de morte no País. ${ }^{6}$

O melhor indicador do impacto da HAS na população são as taxas de doença cerebrovascular, a proporção de mortes por acidente vascular cerebral em relação às demais doenças cardiovasculares, como a DAC e a insuficiência cardíaca. A comparação das taxas brasileiras de mortalidade com a de outros países (europeus e da América Latina) mostra que o risco de morte por doença cerebrovascular é maior no Brasil. ${ }^{7}$ É importante, portanto, frisar que o tratamento antihipertensivo está associado a uma redução de 35\% a 44\% na incidência de acidente vascular encefálico. ${ }^{1}$

Portadores de Diabetes Mellitus (DM) representam pacientes de alto risco para eventos cardiovasculares, os quais continuam sendo a principal causa de morbidade e determinante da mortalidade no contexto do DM. ${ }^{1,3}$ Atualmente, estima-se que a população mundial com diabetes é da ordem de 382 milhões de pessoas. Cerca de $80 \%$ desses indivíduos com DM vivem em países em desenvolvimento, com crescente proporção de pessoas afetadas em grupos etários mais jovens. ${ }^{8} \mathrm{O}$ aumento da incidência e da prevalência do DM2 ocorre em virtude do crescimento e do envelhecimento populacional, da maior urbanização, da crescente prevalência de obesidade e sedentarismo, bem como da maior sobrevida de pacientes com DM.,

A Doença Renal Crônica (DRC) é, atualmente, considerada um problema de saúde pública mundial. No Brasil, a sua incidência e a prevalência estão aumentando. O número projetado atualmente para pacientes em tratamento dialítico e com transplante renal no Brasil está próximo dos 130.000 . Independentemente da etiologia da doença de base, os principais desfechos em pacientes com DRC são as suas complicações (anemia, acidose metabólica, desnutrição e alteração do metabolismo de cálcio e fósforo), decorrentes da perda funcional renal, o óbito (principalmente por causas cardiovasculares) e a necessidade de terapia renal substitutiva (TRS). Estudos recentes indicam que esses desfechos indesejados podem ser prevenidos ou retardados se a DRC for diagnosticada precocemente, e as medidas nefro e cardioprotetoras implementadas imediatamente. ${ }^{10}$

Conforme ensaios clínicos randomizados de grande porte e revisões sistemáticas em pacientes de alto risco cardiovascular, o benefício de fármacos, como as estatinas, estende-se mesmo àqueles com níveis de colesterol normais. Até o presente, a redução do LDL-C por inibidores da hidroximetilglutaril coenzima A (HMG CoA) redutase ou estatinas permanece sendo a terapia mais validada por estudos clínicos para reduzir a incidência de eventos cardiovasculares (CVs). ${ }^{11,12}$ Os antiplaquetários são capazes de reduzir eventos CVs maiores, mesmo em indivíduos sem manifestações clínicas de aterosclerose, e diversos anti-hipertensivos, particularmente os Inibidores da Enzima conversora de Angiotensina (IECA), apresentam potencial para redução de eventos cardiovasculares, até em pacientes sem critérios diagnósticos para HAS. Adicionalmente, intervenções não farmacológicas, como dietas cardioprotetoras, cessação do tabagismo e atividade física, apesar dos dados serem menos robustos do que de estudos com terapia farmacológica, também estão associadas à redução de desfechos clinicamente relevantes. ${ }^{11}$

\section{OBJETIVOS}

Avaliar, em populações de alto risco cardiovascular, a prática clínica vigente na prescrição das intervenções farmacológicas baseadas em evidência, definidas como uso simultâneo de antiplaquetários, estatina, IECA e betabloqueador.

\section{MATERIAL E MÉTODOS}

\section{Metodologia proposta}

Trata-se de estudo observacional, transversal e descritivo 
desenvolvido entre os pacientes atendidos nos ambulatórios de cardiologia do Hospital Universitário Walter Cantídio (HUWC/ UFC), no período de abril de 2014 a maio de 2015 . A população engloba 975 indivíduos de alto risco cardiovascular proveniente de diversas cidades do estado do Ceará, definindo-se como critérios de alto risco aqueles indivíduos que apresentem pelo menos um dos seguintes: doença arterial coronariana (DAC), doença cerebrovascular, doença vascular periférica, diabetes tipo 1 ou 2, doença renal crônica. Foram também considerados como alto risco cardiovascular aqueles que apresentassem pelo menos três fatores de risco para doença aterosclerótica entre os seguintes: hipertensão arterial sistêmica, dislipidemia, idade acima de 70 anos, história familiar de DAC.

Os dados foram coletados de prontuários eletrônicos do Hospital Universitário Walter Cantídio e apresentados em média e desvio padrão ou frequências, quando apropriados. Foram analisadas as seguintes variáveis: faixa etária prevalente, sexo, raça, pressão arterial, fatores de risco cardiovasculares prevalentes (diabetes mellitus, doença arterial coronariana, doença cerebrovascular, doença vascular periférica, doença renal crônica e pacientes que apresentassem pelo menos três dos fatores de risco para doença aterosclerótica listados a seguir: hipertensão arterial sistêmica, tabagismo, dislipidemia, idade acima de 70 anos, história familiar de DAC). Os dados foram analisados através do programa Tableau. Trabalho aprovado pelo comitê de ética. Número do parecer: 1.513.492.

\section{RESULTADOS}

Foram selecionados 833 pacientes do ambulatório de HAS (Hipertensão Arterial Sistêmica), correspondente a 85,4\% do total, e 142 pacientes do ambulatório de DAC- Doença Arterial Coronariana, representando 14,6\% dessa amostra.

As características sociodemográficas estão contempladas nos Gráficos 1 e 2, de onde se pode extrair as seguintes informações:

Gráfico 1. Idade dos pacientes de alto risco cardiovascular - Serviço de Cardiologia do Hospital Universitário Walter Cantídio, 2014-2015.

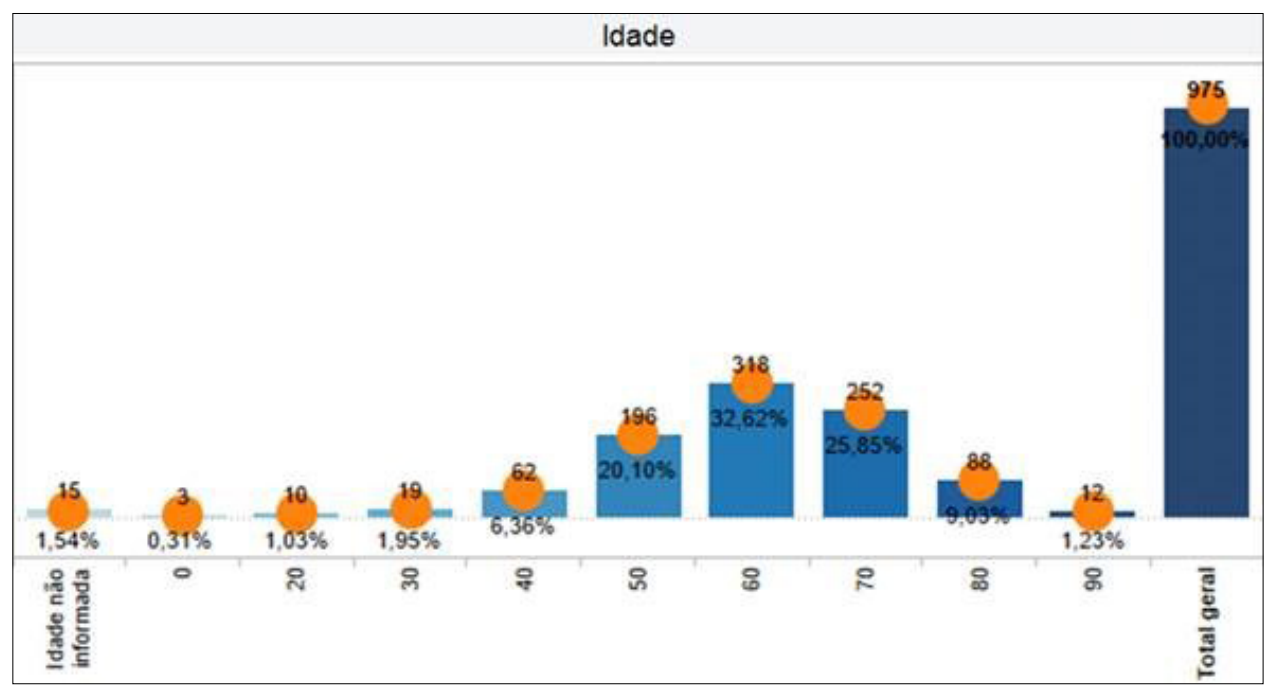

Gráfico 2. Raça dos pacientes de alto risco cardiovascular - Serviço de Cardiologia do Hospital Universitário Walter Cantídio, 2014-2015.

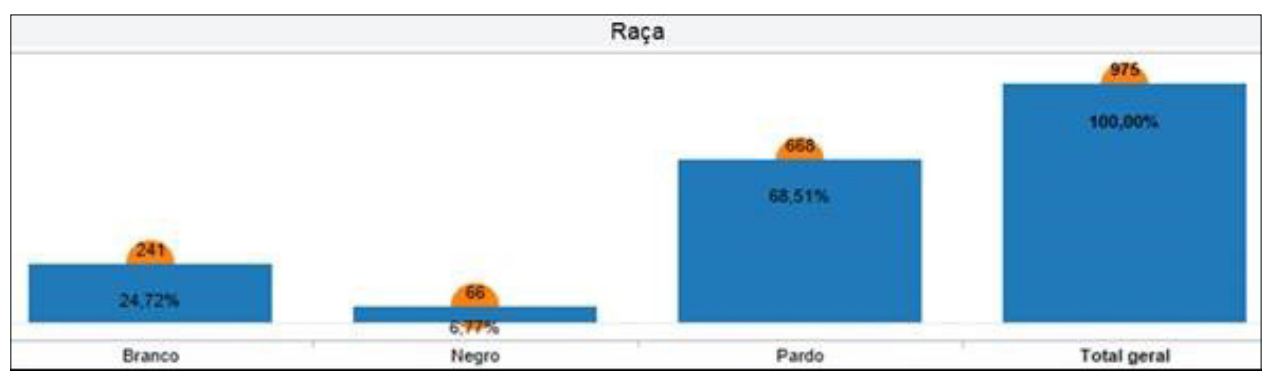

1) a faixa etária prevalente dos pacientes dos ambulatórios de HAS e DAC situou-se entre 50 e 70 anos de idade, respondendo por $78,6 \%$ dos casos, ressaltando, porém, que a preponderância se encontra na faixa etária de 60 anos, que responde por $32,6 \%$ dos pacientes acometidos por HAS e ou
DAC (Gráfico 1). A média de idade calculada foi de 64,8 anos com um desvio padrão de 12,6 anos.

2) $64,7 \%$ eram do sexo feminino; 3 ) $68,5 \%$ eram de cor parda e $24,7 \%$ da raça branca (Gráfico 2). Hipertensão arterial 
sistêmica com 833 pacientes, dislipidemia com 399 e diabetes mellitus com 278 (Gráfico 3) constituíram os principais fatores de risco cardiovasculares.

Da amostra em análise, 631 pacientes $(64,7 \%)$ foram medicados com antiplaquetários, com amplo predomínio de uso do ácido acetilsalić́lico; 613 (62,9\%) com estatinas, principalmente a sinvastatina; 364 (37,3\%) com IECA, destacando-se o captopril; 461 (47,2\%) usaram betabloqueadores, principalmente atenolol, seguido do carvedilol; 595 (61\%) usaram diuréticos tiazídicos, com destaque para a hidroclorotiazida (Gráfico 4). A utilização simultânea de antiplaquetários, estatinas, IECA e betabloqueadores foi constatada em 65 pacientes (Tabela 1), com predominância de uso (40 pacientes) entre os pacientes portadores de DAC, em comparação a outras categorias de alto risco cardiovascular (AVC, diabetes, doença vascular periférica e doença renal). Nenhum paciente com doença vascular periférica (DVP) se encontrava em uso da terapia otimizada, já que nesta é também contemplada o uso de betabloqueadores, que dependendo da gravidade da doença, está contraindicado nesta população.

Gráfico 3. Prevalência de fatores de alto risco cardiovascular e de dislipidemia - Serviço de Cardiologia do Hospital Universitário Walter Cantídio, 2014-2015.

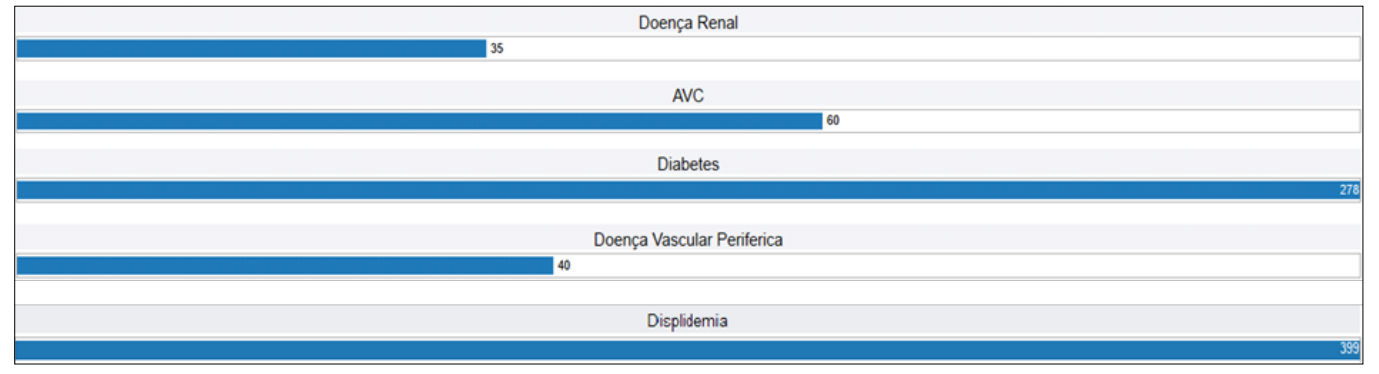

Gráfico 4. Grupos de medicamentos com seu uso de forma isolada nos pacientes de alto risco cardiovascular - Serviço de Cardiologia do Hospital Universitário Walter Cantídio, 2014-2015.

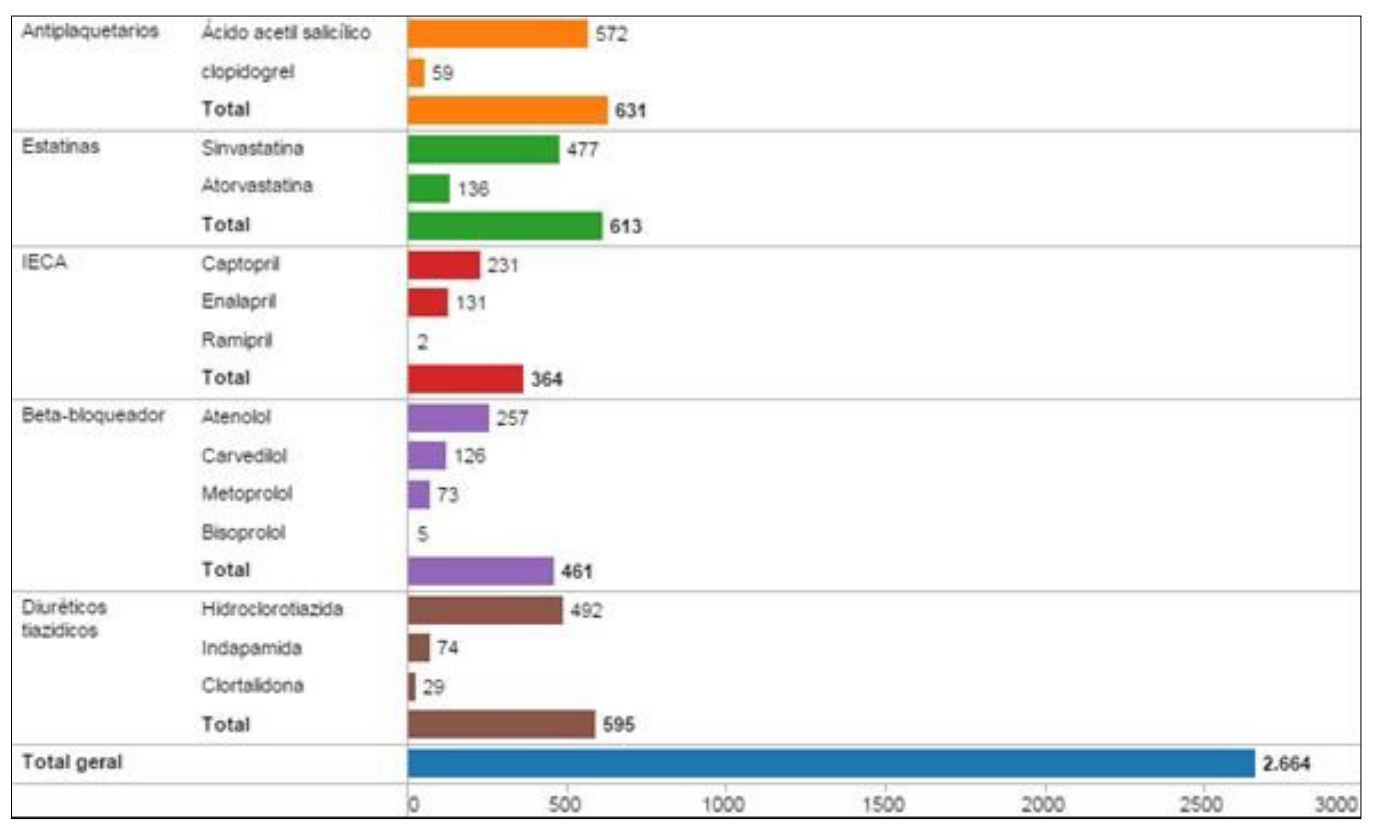

Tabela 1. Fármacos em uso em prescrições para patologias de alto risco cardiovascular - Serviço de Cardiologia do Hospital Universitário Walter Cantídio, 2014-2015.

\begin{tabular}{cccc}
\hline & Estatina + antiplaquetário & Est + antiplaq + IECA & Est + antiplaq + IECA + B-b \\
\hline DAC & $184(54 \%)$ & $55(16 \%)$ & $40(11 \%)$ \\
DM & $144(52 \%)$ & $50(18 \%)$ & $19(6,8 \%)$ \\
AVC & $24(40 \%)$ & $11(18 \%)$ & $5(8,3 \%)$ \\
Doença vascular periférica & $20(50 \%)$ & $7(17,5 \%)$ & 0 \\
Doença renal & $8(22,8 \%)$ & $3(8,5 \%)$ & $1(2,8 \%)$ \\
TOTAL & 338 & 126 & 65 \\
\hline
\end{tabular}


Confrontado com as metas determinadas por diretrizes da American Heart Association (AHA), o estudo evidenciou que, dos pacientes identificados com HAS e ou DAC, $630(64,6 \%)$ apresentavam pressão arterial (PA) sob controle (PA $\leq 140 / 90$ $\mathrm{mmHg})$ e $345(35,4 \%)$ mantinham pressão arterial acima da meta estabelecida tendo como base uma única aferição da pressão arterial.

Entre os pacientes com PA sob controle, foi observado uma média de 120,4 x $77 \mathrm{mmHg}$. Entre os pacientes com PA não controlada, foi observada uma média de 155,9 x 84,2 $\mathrm{mmHg}$.

Pode ser observado ainda que foi obtido um melhor controle da PA entre os portadores de DAC (53\%), seguido pelos pacientes portadores de DM (43,3\%) (Tabela 2).

Tabela 2. Pressão Arterial controlada e não controlada em pacientes de alto risco cardiovascular - Serviço de Cardiologia do Hospital Universitário Walter Cantídio, 2014-2015.

\begin{tabular}{ccc}
\hline & \multicolumn{2}{c}{ Pressão arterial } \\
Doenças & PC & PNC \\
\hline DAC & $181(53 \%)$ & $98(28,9 \%)$ \\
DM & $123(43,3 \%)$ & $90(32 \%)$ \\
AVC & $20(33,3 \%)$ & $20(33,3 \%)$ \\
Doença vascular periférica & $16(40 \%)$ & $11(27,5 \%)$ \\
Doença renal & $05(13,8 \%)$ & $07(19,5 \%)$ \\
\hline
\end{tabular}

PC: pressão controlada; PNC: pressão não controlada.

\section{DISCUSSÃO}

A faixa etária prevalente entre os pacientes diagnosticados com HAS e DAC situou-se entre 50 e 70 anos de idade e é semelhante ao de outros estudos. ${ }^{6,13}$

Hipertensão e doença arterial coronariana prevaleceram no sexo feminino $(64,7 \%)$, entrando em discordância com revisão sistemática quantitativa de 2003 a 2008, de 44 estudos em 35 países, que revelou uma prevalência global de 37,8\% em homens e 32,1\% em mulheres. Tal discrepância pode ser explicada pela maior procura do gênero feminino pelos serviços de saúde. ${ }^{14}$

A maioria dos pacientes era de cor parda $(68,5 \%)$, seguidos por brancos $(24,7 \%)$ e negros $(6,8 \%)$. Lessa e Fonseca, estudando as diferenças raciais no controle da HAS e adesão ao tratamento, verificaram que a frequência de adesão a consultas e tratamento foi significativamente menor em pacientes negros $(19,7 \%)$, em relação aos pardos $(33 \%)$ e brancos $(53,6 \%) .{ }^{15}$ Deve ser levado em consideração que a classificação racial foi julgada pelo examinador e, portanto, pode explicar a discordância entre os resultados dos dois estudos.

Os fatores de risco mais prevalentes na população estudada foram HAS e dislipidemia, o que condiz com a literatura. $\mathrm{O}$ estudo multicêntrico REACH, por exemplo, encontrou prevalência de $81,3 \%$ para HAS e $70,5 \%$ para dislipidemia.
O estudo REACT, por sua vez, reportou prevalência de $92,1 \%$ para HAS e $75,3 \%$ para dislipidemia. ${ }^{11,16}$

No que se refere à prescrição de terapias farmacológicas baseadas em evidências, o estudo em questão evidencia que o uso de antiagregantes plaquetários $(64,7 \%)$ e estatinas $(62,9 \%)$ se aproxima ao de países desenvolvidos. ${ }^{17}$ Tais dados são também semelhantes aos encontrados em outros estudos, como o do REACT que evidenciou taxas de prescrição de $78,3 \% ; 72,6 \%$ e $53 \%$ para antiagregantes plaquetários, estatinas e IECA, respectivamente; e o estudo REACH, que constatou uso de $69 \% ; 72,6 \%$ e $43,3 \%$ para as medicações referidas acima. ${ }^{11,16}$

Estudos clínicos demonstraram que a detecção, o tratamento e o controle da HAS são fundamentais para a redução dos eventos cardiovasculares. ${ }^{1} \mathrm{O}$ presente estudo revelou que a maior parcela da população analisada apresentava valores de PA inferiores a 140/90mmHg (64,6\%); entretanto, 14 estudos populacionais, realizados nos últimos quinze anos com 14.783 indivíduos ( $\mathrm{PA}<140 / 90 \mathrm{mmHg}$ ) no Brasil, revelaram baixos níveis de controle da PA (19,6\%). ${ }^{18,19}$ Não houve diferença significativa no controle da hipertensão arterial entre países desenvolvidos e em desenvolvimento. Entre os homens, o controle da hipertensão arterial se situou em 9,8\% em países em desenvolvimento e 10,8\% em países desenvolvidos. Entre as mulheres esse controle se situou em 16,2\% em países em desenvolvimento e $17,3 \%$ em países desenvolvidos. ${ }^{14}$ Dados ainda inferiores aos obtidos nos estudos populacionais brasileiros e ao do presente trabalho.

Em hypertension-treatment protocol at Kaiser Permanent Northern California, o uso de assistentes médicos treinados e visitas monitorizadas estendidas aumentou a proporção de pacientes nos quais a hipertensão foi controlada, passando de $44 \%$ para $87 \%$ dos pacientes acompanhados, durante um período de 10 anos de seguimento. ${ }^{20}$

Esses dados mostram que os esforços dos profissionais de saúde, em conjunto com as sociedades científicas e agências governamentais, são fundamentais para se atingir metas aceitáveis de tratamento e controle da HAS. ${ }^{1}$

A situação exposta evidencia a necessidade de se reduzir os níveis pressóricos nesta população de risco. Talvez o baixo nível socioeconômico dos pacientes avaliados seja um fator que limite a obtenção de um controle adequado da pressão arterial, apesar de uma parcela importante da população objeto deste estudo receber medicação gratuitamente do Estado. Este fato sugere ou uma falta de aderência ao tratamento ou um descuido dos médicos, em relação ao controle dos níveis pressóricos. Existe uma clara necessidade de se melhorar a abordagem dos pacientes com alto risco para um evento cardiovascular, já que a terapêutica otimizada à base do uso associado de estatinas, betabloqueadores, antiagregantes plaquetários e IECA encontra-se bastante reduzida na população estudada, bem como o uso isolado de tais medicações, reforçando a importância de desenvolvimento de programas de melhoria da prática clínica, através da interação de diversos determinantes, tais como: capacitação 
dos profissionais de saúde, pesquisas envolvendo um maior contingente populacional e em diversos centros pelo Brasil, maior compromisso das agências governamentais referente a recursos materiais e humanos.

Algumas limitações do presente estudo que merecem atenção consistem na aferição da pressão arterial (PA) durante as visitas ao consultório, que consistiu em uma medida isolada de pressão arterial sendo esse o único dado usado como critério de controle ou não da PA; os dados foram obtidos a partir de prontuários eletrônicos e, portanto, dispomos apenas

\section{REFERÊNCIAS}

1. Sociedade Brasileira de Cardiologia; Sociedade Brasileira de Hipertensão; Sociedade Brasileira de Nefrologia. VI Diretrizes Brasileiras de Hipertensão. Arq Bras Cardiol. 2010;95(1 Supl.1):1-51.

2. James PA, Oparil S, Carter BL, Cushman WC, DennisonHimmelfarb C, Handler J, et al. 2014 evidence-based guideline for the management of high blood pressure in adults: report from the panel members appointed to the eighth Joint National Committee (JNC 8). JAMA. 2014;311(5):507-20.

3. Victor RG. Braunwald Tratado de doenças cardiovasculares. Rio de Janeiro:Elsevier; 2013. Parte VI, Capítulo 45, Hipertensão sistêmica: mecanismos e diagnóstico;p. 954.

4. Sipahi I, Tuzcu EM, Schoenhagen P, Wolski KE, Nicholls SJ, Balog C, et al. Effects of normal, pre-hypertensive, and hypertensive blood pressure levels on progression of coronary atherosclerosis. J Am Coll Cardiol. 2006;48(4):833-8.

5. Rosendorff C, Black HR, Cannon CP, Gersh BJ, Gore J, Izzo JL, et al. Treatment of hypertension in the prevention and management of ischemic heart disease: a scientific statement from the American Heart Association Council for High Blood Pressure Research and the Councils on Clinical Cardiology and Epidemiology and Prevention. Circulation. 2007;115(21):2761-88.

6. Pereira JL, Sakae TM, Machado MC, Castro CM. Escore TIMI no infarto agudo do miocárdio conforme níveis de estratificação de prognóstico. Arq Bras Cardiol. 2009;93(2):105-12.

7. Andrade P. Tratado de cardiologia SOCESP. São Paulo: Manole; 2005. Seção5, Capítulo 1, Epidemiologia da hipertensão arterial no Brasil; p. 397-403.

8. International Diabetes Federation. IDF Diabetes Atlas. 6. ed. Belgium: IDF; 2013

9. Sociedade Brasileira de diabetes. Diretrizes da sociedade brasileira de diabetes 2013-2014. São Paulo: AC farmacêutica; 2014.

10. Sociedade Brasileira de Nefrologia; Sociedade Brasileira de Urologia; Sociedade Brasileira de Pediatria; Sociedade Brasileira de Nutrição Parenteral e Enteral. Doença renal crônica (pré-terapia renal substitutiva): diagnóstico - projeto diretrizes 2011. [Acesso em: 16 out 2015]. Disponível em: https://diretrizes.amb.org.br/ BibliotecaAntiga/ doenca_renal_cronica_pre_terapia_renal_substitutiva_diagnostico.pdf do registro de consulta realizada por diferentes médicos os quais, entretanto, foram previamente treinados.

\section{CONCLUSÃO}

Concluímos que a prevalência de HAS e DAC se situou em: pacientes pardos, sexo feminino e idade em torno de 60 anos; os fatores de risco mais prevalentes foram HAS e DLP; o uso da terapia otimizada se encontra aquém da ideal, sendo mais prevalente em portadores de DAC; a maior parcela da população em estudo atingiu a meta para controle de PA.

11. Berwanger O, Mattos LA, Martin JF, Lopes RD, Figueiredo EL, Magnoni $\mathrm{D}$, et al. Prescrição de terapias baseadas em evidências para pacientes de alto risco cardiovascular: estudo REACT. Arq Bras Cardiol. 2013;100(3):212-20.

12. Sposito AC, Caramelli B, Fonseca FA, Bertolami MC, Afiune A Neto, Souza AD, et al. Sociedade Brasileira de Cardiologia. IV Diretriz brasileira sobre dislipidemias e prevenção da aterosclerose. Arq Bras Cardiol. 2007;88(supl 1):1-18.

13. Zornoff LA, Paiva SA, Assalin VM, Pola PM, Becker LE, Okoshi MP, et al. Perfil clínico, preditores de mortalidade e tratamento de pacientes após infarto agudo do miocárdio, em hospital terciário universitário. Arq Bras Cardiol. 2002;78(4):396405.

14. Pereira M, Lunet N, Azevedo A, Barros H. Differences in prevalence, awareness, treatment and control of hypertension between developing and developed countries. J Hypertension. 2009;27(5):963-75.

15. Pierin AM, Nobre F Jr. Risco cardiovascular global - da teoria à prática. São Paulo: Lemos editorial; 2000.

16. Bhatt DL, Eagle KA, Ohman EM, Hirsch AT, Goto S, Mahoney EM, et al. Comparative determinants of 4-year cardiovascular event rates in stable outpatients at risk of or with atherothrombosis. JAMA. 2010;304(12):1350-7.

17. Mendis S, Abegunde D, Yusuf S, Ebrahim S, Shaper G, Ghannem $\mathrm{H}$, et al. WHO study on prevention of recurrences of myocardial infarction and stroke (WHO-PREMISE). Bull World Health Organ. 2005;83(11):820-9.

18. Rosário TM, Scala LC, França GV, Pereira MR, Jardim PC. Prevalência, controle e tratamento da hipertensão arterial sistêmica em Nobres, MT. Arq Bras Card. 2009;93(6):672-8.

19. Jardim PC, Peixoto MR, Monego E, Moreira H, Vitorino PV, Souza WS, et al. Hipertensão arterial e alguns fatores de risco em uma capital brasileira. Arq Bras Card. 2007;88(4):452-7.

20. Jaffe MG, Lee GA, Young JD, Sidney S, Go AS. Improved blood pressure control associated with a large-scale hypertension program. JAMA. 2013;310:699-705.

\section{Como citar:}

Freire JG, Silva RP. Avaliação da terapêutica em pacientes de alto risco cardiovascular. Rev Med UFC. 2019 out-dez;59(4):56-61. 